\title{
Remote Sensing Image Enhancement Based on Orthogonal Wavelet Transformation Analysis and Pseudo-color Processing
}

\author{
Zhiwen WANG ${ }^{1,2,3}$, Shaozi LI ${ }^{1,3 *}$, Yanping $L^{1,3}$, Kaitao YANG ${ }^{1,3}$ \\ ${ }^{1}$ Cognitive Science Department, Xiamen University, 361005 Xiamen,Fujian, P.R. China \\ ${ }^{2}$ Department of Computer \& Engineering, Guangxi University of Technology, 545006 Liuzhou, Guangxi, P.R. China \\ ${ }^{3}$ Fujian key laboratory of the Brain-like Intelligent Systems(Xiamen University), 361005 Xiamen,Fujian ,P.R. China
}

Received: $24-12-2009$
Accepted: $09-09-2010$

Wavelet analysis based on image enhancement technique is only applicable to black-and-white image, and pseudo-color image processing technology cannot adequately deal with some of the details information of the image. In this paper, an enhanced approach of remote sensing image based on orthogonal wavelet analysis and pseudo-color image processing is presented. Enhanced remote sensing image has been greatly improved in both visual effects and noise characteristics. The method is simple yet flexible with less calculation. Moreover, the proposed method also has the advantages of faster computing speed and operating feasibly and so on. It has great potential in research and application of remote sensing image enhancement.

Keywords: Orthogonal wavelet transformation, remote sensing image processing, pseudo-color image processing, image enhancement.

\section{Introduction}

Earth observation satellites provide data called as remote sensing images covering different portions of the electromagnetic spectrum at different spatial, temporal and spectral resolutions. As an advanced detection technology, remote sensing has been widely applied in many areas, such as military reconnaissance, target surveillance, effect assessment of combating or damage, monitoring of crop growth and assessing of grain yield, universal survey of surface resources, and many more. Remote sensing has brought huge economic and social benefits, and shown a broad application prospects. With the development of science and technology, acquisition of high-resolution remote sensing image has become a reality. The processing for remote sensing image has been developed to an all-digital stage, and its applications have been achieved from determining on the nature simply to combining with qualitative and quantification, and it transits from extensive form to fine type. In the whole process of remote sensing, acquisition of remote sensing image is the prerequisite and basis. Remote sensing image processing is a means of application of remote sensing image, and remote sensing image is the purpose and end-result. ${ }^{1,13}$ As the remote sensing image signal will inevitably lose some details in access and transmission, it is essential to enhance remote sensing image. The operations of remote sensing image enhancement include contrast improvement, edge enhancement, color enhancement, nonlinear Stretch, linear enhancement, radiometric enhancement, spatial filtering, noise suppression, image smoothing, and image sharpening, and so on. Image enhancement technology which is based on wavelet analysis for remote sensing image is a newly developed technique., ${ }^{2,7,16}$ In this method, remote sensing images will be converted into a space-frequency domain, and interested part of it will be dealt with space-frequency.

${ }^{*}$ Corresponding authors. Address: Cognitive Science Department, Xiamen University, 361005 Xiamen, Fujian, P.R. China(S.Z. LI)

Tel.:+86 592-2580080.

E-mail:szlig@xmu.edu.cn; 
However, this technique is only applicable to black and white remote sensing images. The pseudo-color processing technology of remote sensing images can convert black-and-white remote sensing images into color remote sensing images, it is also one of traditional image enhancement techniques. It can be used to enhance the resolution of image, because the resolution of color remote sensing images is higher than gray-scale remote sensing images for human eye. However, this technique is unable to adequately deal with some of the details of remote sensing image information. Method for enhancement of remote sensing image based on orthogonal wavelet analysis and pseudo-color processing is proposed in this paper ${ }^{3,8,11}$. In order to improve the accuracy of remote sensing image interpretation, remote sensing images is first treated with wavelet analysis, and then dealt with pseudo-color enhancement when the image detail is strengthened.

The rest of this paper is organized as follows. For completeness, a brief introduction of orthogonal wavelet transformation of image is given in Section 2. Then, Section 3 details the method based on orthogonal wavelet transformation for remote sensing image enhancement. In Section 4, a remote sensing image enhancement processing based on pseudo-color image is described. Section 5 details the method based on orthogonal wavelet transformation and pseudo-color processing for remote sensing image enhancement. After that, simulation results are presented in Section 6 . Finally, this paper is concluded in Section 7.

\section{Orthogonal wavelet transformation of image}

Supposed $S_{i, l}^{0}$ are the series of remaining scale factors of the scale space, $h_{0}$ and $h_{1}$ are coefficients of low-pass filter and high-pass filter of wavelet function, and their modulus are invariant for all scales ${ }^{4,6,12,14,19}$. Rapid decomposition of wavelet transform is shown in Fig. 1.

Where $\left\{\begin{array}{l}s_{i, l}^{j}=\sum_{k, n} h_{0}(k-2 i) h_{0}(m-2 l) s_{k, m}^{j-1} \\ \alpha_{i, l}^{j}=\sum_{k, n} h_{1}(k-2 i) h_{0}(m-2 l) s_{k, m}^{j-1} \\ \beta_{i, l}^{j}=\sum_{k, n} h_{0}(k-2 i) h_{1}(m-2 l) s_{k, m}^{j-1} \\ \gamma_{i, l}^{j}=\sum_{k, n} h_{1}(k-2 i) h_{1}(m-2 l) s_{k, m}^{j-1}\end{array}\right.$

Observed from Fig. 1, edges in one scale of the original image can be decomposed into four components in smaller-scale, which are low-frequency component, high frequency components of the level, vertical and diagonal orientation. They represent different information of the original image respectively, and they are gained through four different filters. $s_{i, l}^{j}$ and $s_{i, l}^{j-1}$ are obtained by rows and columns orientation low-pass filter, and they are information of the next scale outline. $\alpha_{i, l}^{j}$ is gained by $s_{i, l}^{j-1}$ through the row high-pass filter and the column low-pass filter, and it corresponds to the vertical profiles which are mapped by the detail information of the level direction. Similarly, $\beta_{i, l}^{j}$ expresses the level profiles which are mapped by the detail information of the vertical direction $s_{i, l}^{j}$, and $\gamma_{i, l}^{j}$ denotes the detail information of the diagonal direction $s_{i, l}^{j}$.

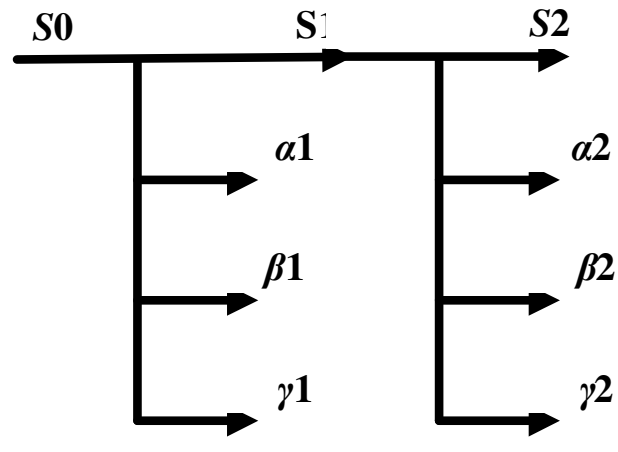

Fig.1. Schematic diagram of orthogonal wavelet transformation

Low-frequency component, high frequency components of the level, vertical and diagonal orientation of image are the results of decomposition of image by using two-dimensional orthogonal wavelet transform. The frame of three layer orthogonal wavelet decomposition is shown in Fig. 2.

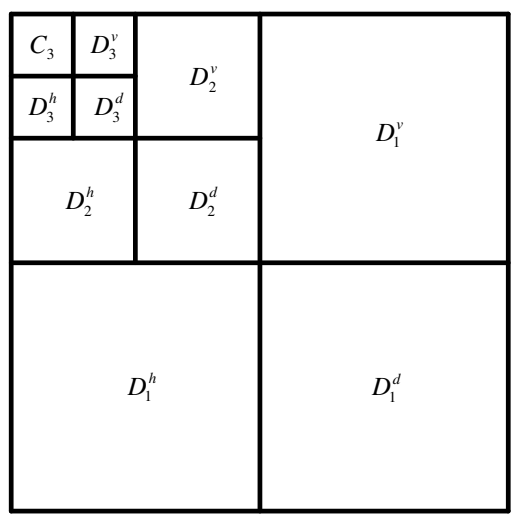

Fig.2. three layer wavelet decomposition of image 
It is low-frequency component of image, and most of image energy is concentrated in this region. $D_{j}^{\lambda}(j=1,2,3 ; \lambda=h, v, d)$ represent level, vertical and diagonal components of image, and they are all the details information of image.

\section{Remote sensing image enhancement processing based on orthogonal wavelet transformation of image}

Remote sensing sensor systems detect reflected or emitted radiation from features on the Earth's surface. Often times, the detected energy does not fill the entire grey level range the sensor is capable of. For example, an image containing water, forest and agriculture may have a range of 183 different grey levels. However, the sensor may actually be capable of recording 256 different grey levels ( 8 bit system). Some sensors can be sensitive to tens of thousands, millions or billions of grey levels. Remote sensing image enhancement based on orthogonal wavelet transformation can redistribute these values to utilize the full range of a display. ${ }^{21}$ Wavelet coefficients of corresponding position in all levels of remote sensing image signal often have a strong correlation, but wavelet coefficients of noise has the characteristics of weak correlation or non-relation. We introduce orthogonal wavelet transform to enhance remote sensing image in this paper. To enhance the remote sensing image, firstly, the remote sensing image signals are processed by using wavelet fractal interpolation means, and better noise elimination can be gained. Secondly, an image is decomposed into components whose size, location and direction are different by using orthogonal wavelet transform. Finally, some coefficients of orthogonal wavelet transform are changed before being carried out inverse wavelet transform, so that we can choose to enlarge some weights in which we are interested and decline some components which are unimportant for our research. Wavelet analysis method is an analysis method in which frequency window and space window can be adaptively changed, and space-frequency can be localized ${ }^{3,10,11}$ as well. It has the better characteristics of space-frequency window than traditional Fourier analysis methods.

Supposed $\Phi(x)$ is scaling function of onedimensional wavelet analysis, $\Psi(x)$ is corresponding wavelet function, the relationship between twodimensional scaling function and two-dimensional orthogonal wavelet function can be defined as follows: $4,9,15$

$$
\left\{\begin{array}{l}
\Phi(x, y)=\Phi(x) \cdot \Phi(y) \\
\Psi^{1}(x, y)=\Phi(x) \cdot \Psi(y) \\
\Psi^{2}(x, y)=\Psi(x) \cdot \Phi(y) \\
\Psi^{3}(x, y)=\Psi(x) \cdot \Psi(y)
\end{array}\right.
$$

Where $\Phi(x, y)$ is two-dimensional scaling function, $\Psi^{1}, \quad \Psi^{2}$ and $\Psi^{3}$ are three two-dimensional orthogonal wavelet functions.

Two-dimensional multi-scale discrete orthogonal wavelet analysis is defined as following:

$$
\left\{\begin{array}{l}
S_{j} f(n, m)=\iint_{R^{2}} f(x, y) 2^{2 j} \Phi_{j}\left(x-2^{-j} n, y-2^{-j} m\right) d x d y \\
W_{J}^{1} f(n, m)=\iint_{R^{2}} f(x, y) 2^{2 j} \Psi_{j}^{1}\left(x-2^{-j} n, y-2^{-j} m\right) d x d y \\
W_{j}^{2} f(n, m)=\iint_{R^{2}} f(x, y) 2^{2 j} \Psi_{j}^{2}\left(x-2^{-j} n, y-2^{-j} m\right) d x d y \\
W_{j}^{3} f(n, m)=\iiint_{R^{2}} f(x, y) 2^{2 j} \Psi_{j}^{3}\left(x-2^{-j} n, y-2^{-j} m\right) d x d y
\end{array}\right.
$$

Where $f(x, y)$ is remote-sensing image signal, $S_{J} f(n, m)$ is a low-frequency components of $f(x, y)$. $W_{J}^{1} f(n, m), W_{j}^{2} f(n, m)$ and $W_{j}^{3} f(n, m)$ represent the vertical, diagonal and horizontal high-frequency components of $f(x, y)$, respectively. ${ }^{4}$

Thus, remote-sensing image is divided into low frequency components and vertical, diagonal and horizontal high-frequency components after we execute two-dimensional discrete orthogonal wavelet. Thereinto, low-frequency components represent the contours of remote sensing image, while high-frequency components express the details of remote sensing image. As the low-contrast remote sensing images are mainly manifested that three high-frequency components of images are too small, and result in blurred detail of image, so we can enhance the detail information of image by stepping up three high-frequency components remote sensing images. Thus, when the equation (4) was established, namely, the weight of high-frequency components of remote sensing image is larger, the remote sensing image looks clearer.

$$
C_{j}^{\prime}=\left\{\begin{array}{l}
k \times C_{j}^{H}, k>1 \\
C_{j}^{L}
\end{array}\right.
$$

Here, $C_{i}^{H}$ is high-frequency wavelet coefficients of remote sensing images, $C_{j}^{L}$ is low-frequency wavelet coefficients of remote sensing images, $k$ is high- 
frequency component weights of remote sensing images, $j$ is wavelet decomposition level of remote sensing images.

However, this method may cause increasing the high-frequency components or reducing the lowfrequency components of remote sensing image. Images gained are often partially bright or partially obscure with poor contrast, so the image resolution needs to be further enhanced by using pseudo-color processing.

\section{Remote sensing image enhancement processing based on pseudo-color image processing}

In this section, remote sensing images are processed by using frequency domain enhancement method of pseudo-color image. Pseudo-color image enhancement algorithm is described on the basis of the RGB color space. This algorithm does not consider the luminance component independently. Therefore, even if colors are properly assigned, intensity values are randomly changed. This algorithm can be described with four steps. Firstly, black and white remote sensing image is transformed into frequency domain by using Fourier transform, and then separated into three independent components by three filters whose transfer characteristics are different in the frequency domain. Secondly, they are processed by using inverse Fourier transform, three monochrome images which represent different frequency components will be gained, and then three images will be processed further (such as histogram equalization). Finally, they are added to the color display of red, green and blue display channels as tri-phosphor components respectively, and then pseudocolor enhancement of frequency domain subsection is achieved. The block diagram is shown in Fig. 3.

\section{Remote sensing image enhancement based on orthogonal wavelet transformation and pseudo-color processing}

In order to overcome the shortcomings of orthogonal wavelet transformation remote sensing image enhancement algorithm and remote sensing image enhancement method based on pseudo-color processing, an enhanced approach of remote sensing image based on orthogonal wavelet analysis and pseudo-color image processing is presented in this paper. This algorithm can

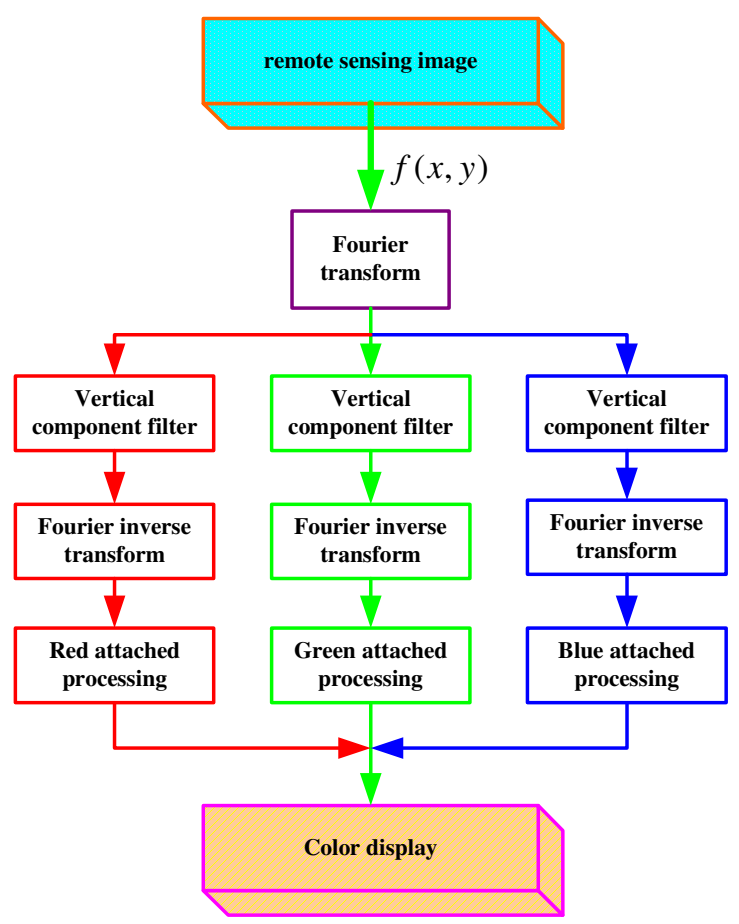

Fig.3. Remote sensing image enhancement diagram by using pseudo-color processing method

overcome the defects of partial light image and poor contrast in the course of remote sensing image enhancement processing by using orthogonal wavelet transform, due to the high and low frequency components of remote sensing image are enhanced simultaneously. At the same time, it can overcome the deficiencies that some detail information of the remote sensing image can not be adequately addressed by using remote sensing image enhancement based on pseudocolor processing. The specific implementation of the algorithm is as follows:

Step 1: firstly, the remote sensing image signals are processed by using wavelet fractal interpolation means, and better noise elimination can be gained.

Step 2: remote sensing image is decomposed into low-frequency component and horizontal, vertical, diagonal high-frequency components whose size, location and direction are different by using orthogonal wavelet transform.

Step 3: in order to achieve image enhancement purposes, the interested components of remote sensing image are zoomed selectively and the components of remote sensing image which take less impact on the results of image are weakened. 
Step 4: The remote sensing image is dealt with by using remote sensing image enhancement method based on pseudo-color processing according with its frequency domain after enhanced by using orthogonal wavelet transform image enhancement approach. Thus, we can get better remote sensing image.

\section{Experimental results and analysis}

The method proposed above has been simulated with Matlab 7.0. The results are shown in Fig. 4. Fig.4 (a) is the original remote sensing image of an area, the background of image is darker, and some areas are unclear. Fig.4(b) is enhanced image by using traditional pseudo-color enhancement approach. Although the resolution of image has been increased, the color effect of the color similar regional does not differentiate well, due to gray values have lesser difference in some places. Fig.4(c) is enhanced image by using orthogonal wavelet analysis means, its resolution is higher than the original image, but image enhancement effect is still less ideal. Fig.4(d) is enhanced image by using method proposed in this paper. It can be easily observed that level of sense of Fig.4(d) is stronger than Fig.4(c).

When the remote sensing image is processed by using orthogonal wavelet analysis, decomposition levels $L$ of orthogonal wavelet need to be as many as possible for containing rich high-frequency coefficients of the details. Selection of weight $k$ of high-frequency components is determined by image characteristics. Value of $k$ should not be too small, otherwise the image details are not abundant. However, in order to avoid introducing noise, Value of $k$ should not be too large either. In experiment of this paper, we choose wavelet function sym4, decomposition levels $L=3$, and $k=5$.

There may be some vague details in remote sensing images, because the images acquired by satellite remote sensing are influenced by a number of effects based on the carrier frequency of the electromagnetic waves and many interfering factors in the course of images capturing, such as exposure and photographic density, defocus of imaging system, diffraction effect, highfrequency loss of image caused by atmospheric disturbances, noise, etc. There are blue dark shadow in remote sensing image which makes the image blurred (shown in Fig. 5.), because the sunlight in the field of image for remote sensors to obtain is scattering in the atmosphere.

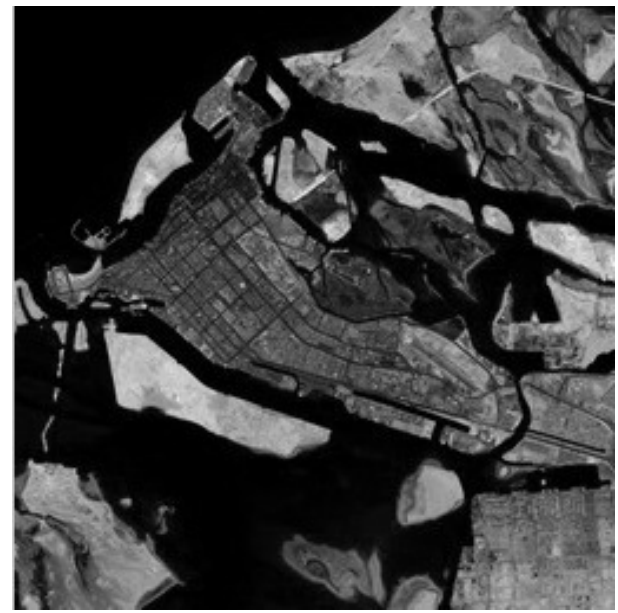

(a) Original image

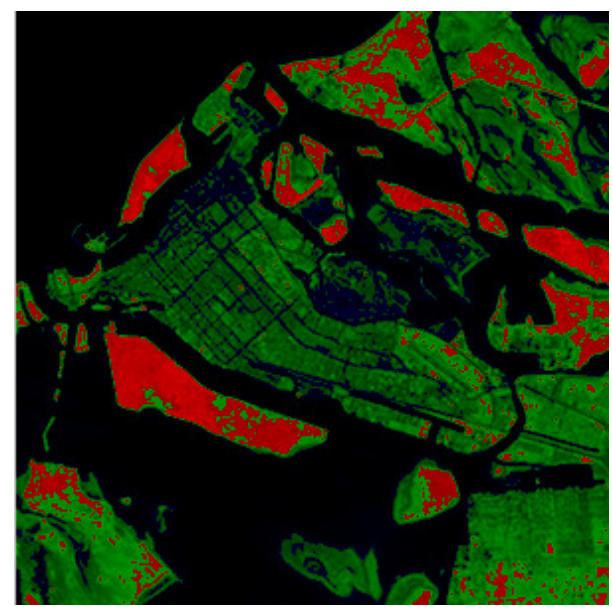

(b) Enhanced image by using traditional pseudo-color enhancement approach

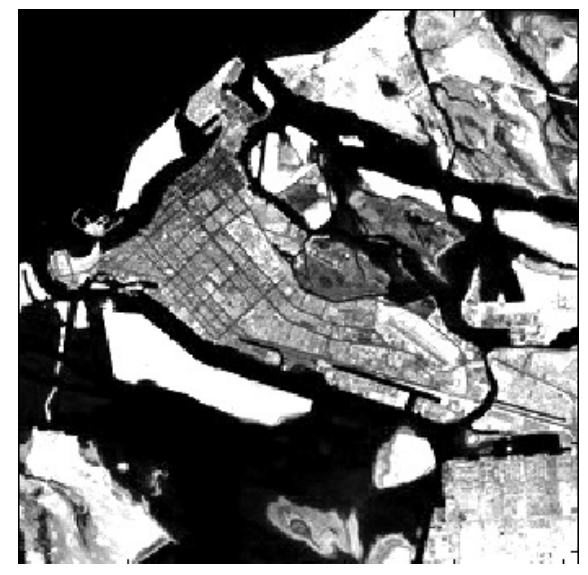

(c) Enhanced image by using wavelet transformation method 


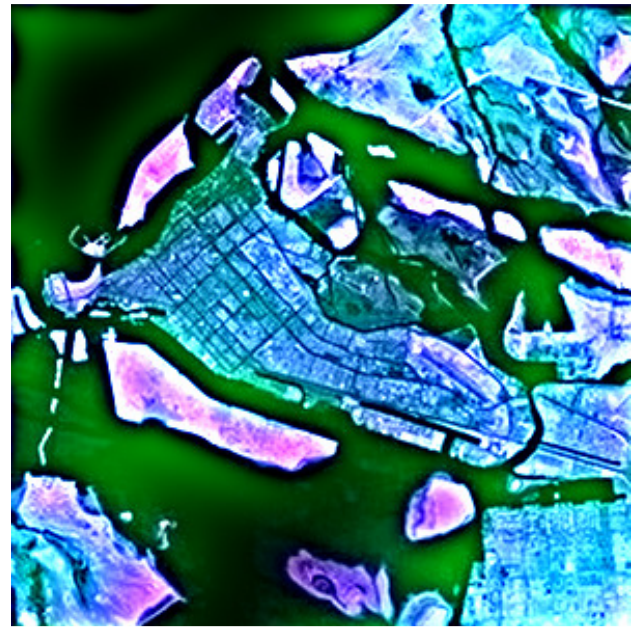

(d) Enhanced image by using method proposed in this paper

Fig.4. Comparison of enhanced image

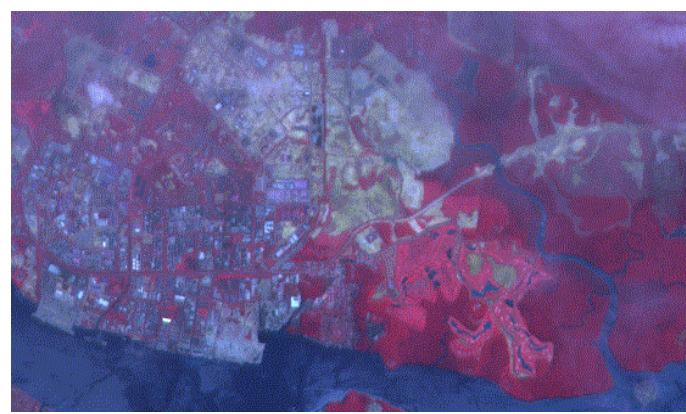

Fig.5. Original remote sensing image

In order to enhance the image by using the method proposed in this paper, we first classify the image according to its spectral characteristics. Classification is shown in Fig. 6. The classification accuracy of remote sensing images is improved when multiple source image data are introduced to the processing. Images from microwave and optical sensors offer complementary information that helps in discriminating the different classes. An overview on image enhancement techniques for change detection from multi-sensor data including image differencing, rationing and PCA is given in Greve Clifford W. et al. (1993). And then, the remote sensing images are processed according to the algorithm described in section 3 and 4. After that the image is enhanced by using the proposed method, the enhanced image is shown in Fig. 7.

Quantitative effectiveness of enhanced remote sensing image is estimated by calculating the mean

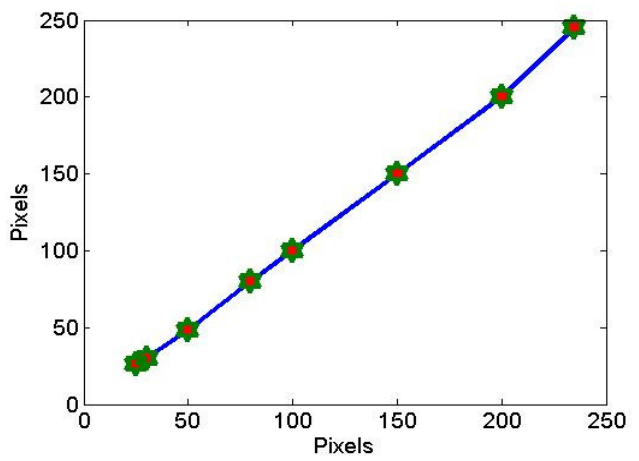

(a) The class diagram for the average pixel value between near-infrared bands and red bands

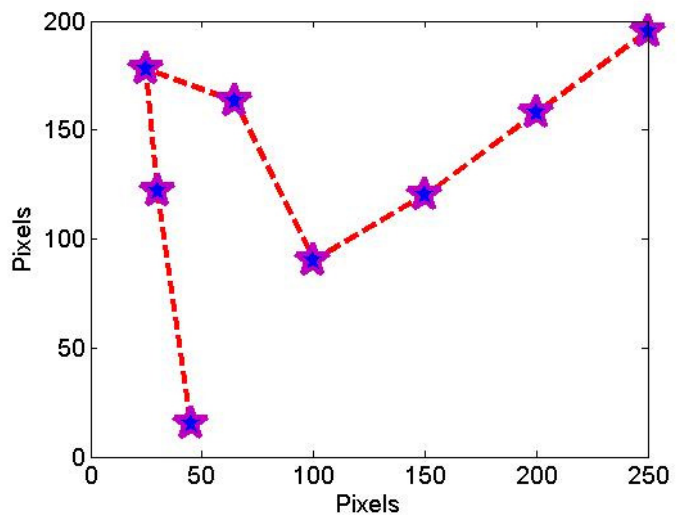

(b) The class diagram for the average pixel value between green bands and red bands

Fig.6. The class diagram for the average pixel value of original remote sensing image

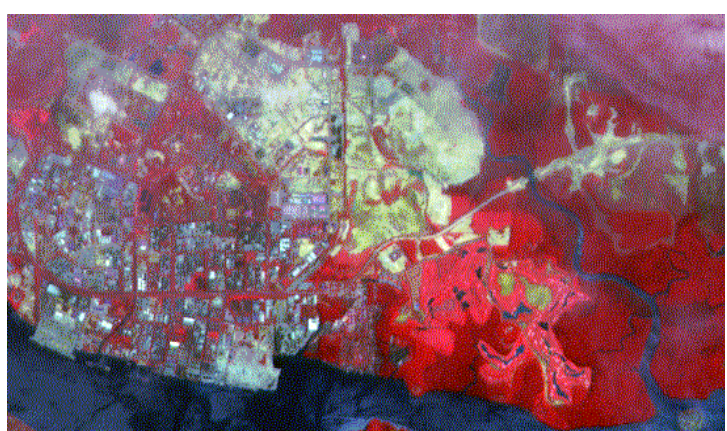

Fig.7. Enhanced image by using method proposed in this paper

value, information entropy and definition of remote sensing images. The mean value of remote sensing images is calculated by using equation (5). 


$$
\text { mean }=\frac{1}{X Y} \sum_{x=1}^{X} \sum_{y=1}^{Y} I_{e}(x, y) \text {. }
$$

Where, $I_{e}$ is enhanced image, $(X, Y)$ is the size of remote sensing image. For a remote sensing image, the mean reflects average brightness of remote sensing images. If mean is moderate (gray value in the vicinity of 128), it indicates good visual effects.

Calculation of information entropy is defined as follows:

$$
E n t=-\sum_{i=0}^{L-1} p_{i} \operatorname{In}\left(p_{i}\right) .
$$

Where, $p_{i}$ is the probability of Remote sensing images that gray-scale $G N L_{i}$ corresponds to. $L$ is the total number of gray-scale. When the Entropy value is higher, the more information can be carried by remote sensing images. Therefore, information entropy is an important indicator which can measure the abundant degree of information of remote sensing image ${ }^{18,20}$.

Definition of remote sensing image can be calculated by using equation (7):

$$
\left\{\begin{array}{l}
\operatorname{Def}=\frac{1}{\mathrm{XY}} \sum_{x=1}^{\mathrm{X}} \sum_{y=1}^{\mathrm{Y}} \sqrt{\left(\Delta I_{x}(x, y)\right)^{2}+\left(\Delta I_{y}(x, y)\right)^{2}} \\
\Delta I_{x}(x, y)=I_{e}(x, y)-I_{e}(x-1, y) \\
\Delta I_{\mathrm{y}}(x, y)=I_{e}(x, y)-I_{e}(x, y-1)
\end{array}\right.
$$

Definition of remote sensing image can reflect the tiny detail and texture transformation characteristics of image. Greater definition of remote sensing image is, indicating the corresponding image clearer. We selected 100 remote sensing images as test images processed by using the method proposed in this paper and traditional pseudo-color enhancement approach and orthogonal wavelet analysis algorithm. The mean value, information entropy and definition of remote sensing image enhanced by using three kinds of algorithms are shown in Tab.1, the relation between information entropy and definition is shown in Fig.8, while the mean value, information entropy and definition of each RGB channel of enhanced color remote sensing image which is processed by using three kinds of algorithms are shown in Tab.2.

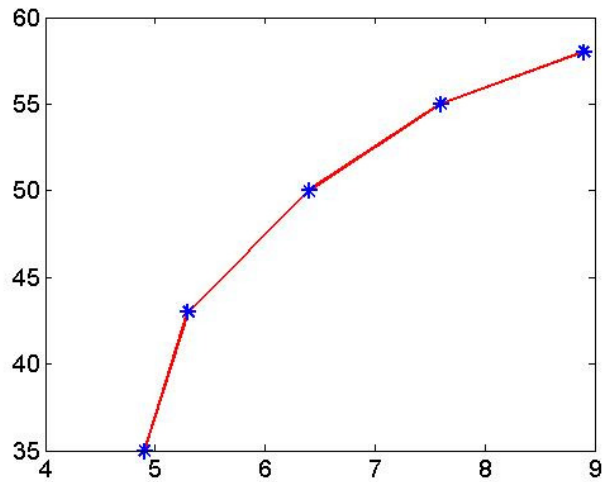

Fig.8. The curve chart of the relation between information entropy and definition

Observed from the experimental results, visual effect of enhanced panchromatic remote sensing image which is processed by using algorithm proposed in this paper is good. Mean value is 132, the information entropy is $15 \%-52 \%$ higher than pseudo-color enhancement algorithm and about $20 \%$ higher than orthogonal wavelet transform domain algorithms. The definition of algorithm is $30 \%-60 \%$ higher than pseudocolor enhancement method, and is about $17 \%$ higher than orthogonal wavelet transforming domain algorithm. Visual effect or noise characteristics of enhanced remote sensing images have been improved greatly.

Table 1. Mean value, information entropy and definition of enhanced black and white remote sensing images

\begin{tabular}{lccc}
\hline Method & $\begin{array}{l}\text { Traditional } \\
\text { enhancement approach }\end{array}$ & $\begin{array}{l}\text { Orthogonal wavelet } \\
\text { analysis means }\end{array}$ & $\begin{array}{l}\text { Method proposed } \\
\text { in this paper }\end{array}$ \\
\hline Mean value & 105 & 95 & 132 \\
$\begin{array}{l}\text { Information } \\
\text { entropy }\end{array}$ & 4.9 & 5.3 & 6.4 \\
Definition & 35 & 43 & 50 \\
\hline
\end{tabular}


Table 2. Mean value, information entropy and definition of enhanced color remote sensing images

\begin{tabular}{|c|c|c|c|c|c|c|c|c|c|}
\hline \multirow[t]{2}{*}{ method } & \multicolumn{3}{|c|}{$\begin{array}{l}\text { Method of Pseudo-color } \\
\text { image enhancement }\end{array}$} & \multicolumn{3}{|c|}{$\begin{array}{c}\text { Remote sensing image } \\
\text { enhancement based on } \\
\text { orthogonal wavelet transform }\end{array}$} & \multicolumn{3}{|c|}{$\begin{array}{c}\text { Remote sensing image } \\
\text { enhancement by using methoo } \\
\text { proposed in this paper }\end{array}$} \\
\hline & $\mathrm{R}$ & $\mathrm{G}$ & $B$ & $\mathrm{R}$ & $G$ & $B$ & $\mathbf{R}$ & $\mathbf{G}$ & B \\
\hline $\begin{array}{l}\text { Mean } \\
\text { value }\end{array}$ & 105 & 96 & 92 & 85 & 76 & 76 & 130 & 122 & 109 \\
\hline $\begin{array}{l}\text { information } \\
\text { entropy }\end{array}$ & 5.0 & 4.9 & 4.8 & 5.2 & 5.1 & 5.1 & 5.6 & 5.5 & 5.5 \\
\hline Definition & 30 & 29 & 29 & 36 & 35 & 35 & 45 & 44 & 44 \\
\hline
\end{tabular}

\section{References}

\section{Conclusion}

Image enhancement technique based on wavelet analysis is only applicable to black-and-white image, and pseudo-color image processing technology cannot adequately deal with some of the information details of the image. To overcome these problems, an enhanced approach of remote sensing image based on orthogonal wavelet analysis and pseudo-color image processing is presented in this paper. Comparison of the scheme proposed in this paper has been done with two other well known techniques based on orthogonal wavelet transformation and pseudo-color image processing. Experimental results have shown that the performance of the algorithm proposed in this paper is superior to that of orthogonal wavelet analysis and pseudo-color image processing based one. Proposed remote sensing image enhancement approach based on orthogonal wavelet analysis and pseudo-color is easy to actualize, and the amount of calculating is small, and its application is more flexible. This method also owns characteristics of fast computing speed and strong operability and so on. In remote sensing image enhancement, it has broad application prospects.

\section{Acknowledgements}

The authors are very grateful for the support provided by National Nature Science Foundation of China (No.60873179), Doctoral Program Foundation of Institutions of Higher Education of China (No. 20090121110032), and Shenzhen Science and Technology Research Foundation (No.JC200903180630A, ZYB200907110169A). (60873179) and the Natural Science Foundation of Guangxi Education Department (200707LX196, 200707LX182).
1. Z.W. WANG, M.Z. LIU and Q.X. CAI, Remote Sensing Image Boosting Based on Wavelet Analysis and Fake Chromatic Processing, Computer Engineering \& Design. 29(18) (2008) 4771-4772.

2. DO.M.N and VRTTERI. I. M, The contourlet transform: an efficient directional multi-resolution image representation, IEEE Trans. on Image processing. 14(12)(2005)2091-2106.

3. Z.W. WANG, M.Z. LIU and Q.X. CAI, Image Fusion Algorithm Based on Weighted and Improved Wavelet Transform, Computer Engineering. 35(11)(2009)228-230.

4. J.A. Antonino-Daviu, M. Riera-Guasp and M. PinedaSanchez, et al, Feature Extraction for the Prognosis of Electromechanical Faults in Electrical Machines through the DWT, International Journal of Computational Intelligence Systems. 2(2)(2009)158-167.

5. M. A. Chaudhry, M. Mufti and M. N. Jafri, Optimal Design of Orthogonal Wavelet Bases for Texture Discrimination Based Upon Fisher Criterion, in Proceedings 17th 2005 International Conference on Microelectronics, ICM, (Islamabad, Pakistan 2005), pp. 155-159.

6. M. Unser, P. Thevenaz and A. Aldroubi, Shift-orthogonal wavelet bases, IEEE Transactions on Signal Processing. 46(7)(1998) 1827-1836.

7. S. L. Linfoot, Wavelet families for orthogonal wavelet division multiplex, Electronics Letters. 44(18)(2008) 1101-1102.

8. S. j. Fu, Q.Q. Ruan and W. Q. Wang, Feature-oriented fuzzy bidirectional flow for image enhancement, Chinese Optics Letters. 4(3) (2006)155-157.

9. Z. H. ZHANG, K. J. LI and J. Y. GAO, Research and Implementation of Algorithm for Image Enhancement and Unwrapped Distortion Correction for SLVF Panoramic Night Vision Image, Journal of Beijing Institute of Technology. 17(4) (2008)423-428.

10. S. J. FU, Q. Q. RUAN, and W. Q. Wang, Region-based Anisotropic Diffusion with Soft Shock Filter for Adaptive Image Enhancement,Chinese of Journal Electronics. 17(1) (2008)56-58.

11. W. Wei and J. Sun. COLOR AC-PDP ENHANCEMENT OF LOW LEVELS IMAGE QUALITY WITH DYNAMIC MODIFYING SUB-FIELDS CODE, 
Academic Journal of Xi'an Jiaotong University. 18(2) (2006)126-130.

12. M. H. XIE and Z. M. WANG, Anisotropic nonlinear diffusion approach to single image resolution enhancement, Opto-electronics Letters. 1(2)(2005)144147.

13. L. W. Chang and Y. E. Shen, Numerical solutions for orthogonal wavelet filters by Newton method, Signal Processing: Image Communication. 14(10)(1999) 879887.

14. P.L. Levin, M. Spasojevic and R. Schneider, Creation of sparse boundary element matrices for 2-D and axisymmetric electrostatics problems using the biorthogonal Haar wavelet, IEEE Transactions on Dielectrics and Electrical Insulation. 5(4)(1998)469-484.

15. T. Li, and S. I. Kamata, Image enhancement by analysis on embedded surfaces of images and a new framework for enhancement evaluation, IEICE Transactions on Information and Systems. E91-D(7)(2008)1946-1954.

16. S. M. Pandit and G. A. Joshi, Image enhancement: A data dependent systems approach, Journal of Engineering for Industry. 116(2)(1994)247-252.
17. C.W. Greve, J.A. Kelmelis and R. Fegeas et al, Investigating U.S. geological survey needs for the management of temporal GIS data, Photogrammetric Engineering and Remote Sensing. 59(10)(1993)15031507.

18. C. POHL and J. L. VAN GENDEREN, Multisensor image fusion in remote sensing: concepts, methods and applications, Int. J. Remote Sensing. 19(5)(1998) 823-854.

19. J. A. Benediktsson, M. Pesaresi, and K. Arnason, Extraction for Remote Sensing Images from Urban Area Based on Morphological Transformations, IEEE Transactions on Geoscience and Remote Sensing. 41(9) (2003)1940-1949.

20. d. W. Valérie, S. Stefan and E. K. Etienne, New Vector Ordering in the RedGreenBlue Colour Model with Application to Morphological Image Magnification, International Journal of Computational Intelligence Systems. 1(2)(2008)103-115.

21. Glossary of remote sensing terms, http://www.ccrs.nrcan.gc.ca/glossary/index_e.php?id=28 89. 